\title{
The Concept of the Mathematical Infinity and Economics
}

\author{
Bhekuzulu Khumalo \\ Private Researcher, Toronto, Canada \\ Email: bhekuzulu.khumalo@gmail.com
}

Received June 4, 2012; revised July 3, 2012; accepted July 10, 2012

\begin{abstract}
Mathematics is the basis of all science for the simple fact that it allows us to measure, counting in its basic sense is measuring. Mathematics is most useful when it is accurate. When we look at the concept of infinity we get new insights into mathematics and how it can be more accurate. This paper endeavors to show that understanding infinity will lead scientists, including economists to take into consideration another classification of variables over and above the traditional classification of continuous and discrete variables. This classification is the dimension of the variable. This problem would never have come to light if knowledge was not given a unit, the knowl, giving anything a unit allows it to be studied in a scientific manner. One finds that knowledge behaves as if it is a three dimensional variable and at other times as if it has infinite dimensions, and the mathematics has to be modified to deal with knowledge as it behaves differently. The reasons are explained hopefully fully in this paper to be grasped and understood. This paper is a follow up to a research note published in International Advances in Economic Research, titled "The Concept of the mathematical Infinity and Economics".
\end{abstract}

Keywords: Variable; Dimension of Variable; Derivative of Three Dimensional Variable; Continuous/Discrete Variable; Trans Dimensional Mathematics; Marginal Analysis

\section{Is It Obvious}

[1] "On a drizzly afternoon in 1886, Camille Jordan entered a small building behind the Pantheon in Paris to deliver a lecture to his mathematics class at the Ecole Polytechnique ... He intended to prove a theorem by a means of a statement that he had always thought obviously true, so he casually relayed it to the class. A vigilant student, seated in the last row, politely interrupted the great professor to ask for more evidence or a proof of what was claimed to be 'obvious'. Professor Jordan scratched his head, stroked his beard, and rapidly blinked his eyes as he nervously removed his wire rimmed glasses from one ear at a time and thought about how he would convince the class that the simple statement he had made was, indeed, true. After pondering the statement more carefully for several minutes without saying a word, he concluded that perhaps it was not so obvious." (Mazur)

We will find in this paper that what is obvious needs logical and philosophical arguments, though the truth can be considered obvious. Further quoting from Joseph Mazur [1], "Jordan initially thought his statement was obvious. What could that mean? I suppose he thought it required no thought or consideration for the mind to accept it. Perhaps he initially thought it hard not to easily sense its truth. To him, its truth was clear and apparent, as if he could sense it with his own eyes. But even truths that are seen through the eyes can be called into question. When Galileo discovered four new moons orbiting Jupiter, he was admonished because he had observed them through a telescope and had not deduced them from logical arguments. Here is a case in which someone is seeing the moons of Jupiter and is told what he is seeing cannot be true because logical argument is better than direct observation."

In a manner of speaking humans do not want their truths challenged and find any excuse for the status quo. What do numbers represent, what is the basis of numbers? Numbers are the basis of counting, without numbers we cannot count. A number according to the dictionary is a "word or symbol, or a combination of words or symbols, used in counting or in noting a total." We need to be clear what counting is, the dictionary defines counting as, "to check over (the separate units or groups of a collection) one by one to determine the total number; add up; enumerate: He counted his tickets and found he had ten." Therefore a number is a word or symbol used for counting, and counting is done in order to determine a total number.

Numbers are the basis of counting, counting that is done in order to determine a total number, meaning that counting is the basis of measuring. It follows that we cannot measure without counting and we cannot count 
without numbers. Being able to count, 2 cups, 3 baskets, 6 antelope, we are basically measuring. Every culture, humans to survive need a basic pattern to recognize a way to measure, two moons ago, my stomach is full because I ate so much of an antelope. Then a human because they can measure will understand portions, not the whole leg, but if you divide that leg into 5 equal parts, one of those parts will fill me up. From counting we can understand portions, very important because how else would humans share and reward each other. A portion is a measurement.

Measurement is the basis of scientific thought, so much iron plus so much titanium and the alloy will be so strong, GNP is equal to so much is basically a tally, counting, what others think as more sophisticated methods of counting, but counting nonetheless. Counting is counting be one using fingers or fancy notation. One sitting in an air conditioned office in downtown Manhattan counting billions of dollars in revenue is no different from a Babylonian merchant, or Mwenemutapa merchant in ancient times counting his revenue, or a Cavemen counting an antelope herd, they are all taking a tally, it is just counting.

Given that numbers arose from our need to measure, once they existed they existed. It is when people settled down that they had time to do something more with numbers. Quoting from Dirk Struik [2], "little progress was made in understanding numerical value and space relations until the transition occurred from the mere gathering of food to its actual production, from hunting and fishing to agriculture ... Fisherman and hunters were in large part replaced by primitive farmers. Such farmers remaining in one place as long as the soil was fertile, began to build more permanent dwellings ..." This is not a paper on mathematical history, but hopefully one understands that with settlements people had time to look more carefully at the numbers and find relationships between numbers, multiplication instead of addition, then division, things that nomads would not have for example found the time for.

With more understanding of relationships the notation could become more abstract, but was accepted as long as it was logically sound. Take the relationship $a+b=c$. It is just a relationship and breaks no laws of logic or mathematics. $a$ and $b$ can be any numbers, and their sum is equal to c. But though very abstract, the relationship still represents just counting, one is tallying $a$ and $b$.

All mathematical relationships essentially, in one way or another, represent measuring, $\pi \mathrm{r}^{2}=\mathrm{A}$ is the area of a circle, this is a relationship between $\pi$ and $\mathrm{r}$, where $\pi$ is pi, $\mathrm{r}$ is the radius of the circle and $\mathrm{A}$ is the area of the circle. $\mathrm{R}$, the radius of the circle, is not limited, it can be $1 \mathrm{~cm}$ or 1 billion $\mathrm{km}$, but we can get to the area because we understand the relationship that determines the area of a circle. It is here for example that we enter into the dilemma of infinity. The area of a circle is being used here because it is assumed that anybody capable of reading and deciphering this paper was taught this relationship in their early teenage years. But first let us make sure we understand the concept of infinity in the mathematical sense.

\section{Cantor's Logic}

[3] "It was Georg Cantor, a Russian-German mathematician, who resolved these seeming paradoxes that cir cled the notion of infinity. It was a brilliant piece of work. He defined infinity through infinite collections. Such col lections were characterized by the fact that you could subtract a finite number of elements from them without changing the size of the collection. You could even subtract an infinite number ..." (Kaplan)

Cantor's logic was unique and correct, this paper does not seek in any manner to even suggest Cantor was wrong, he was right. The paper merely seeks to make clear the application of Cantor's logic in the practical rather than in the abstract where Cantor's logic remains. Cantor's logic is great even today [3], "This proof-as simple and subtle as all great art-throws open the gates to what Hilbert called Cantor's paradise." (Kaplan). Being simple it is easy to understand.

The application of Cantor's logic outside the abstract can be found in Cantor's thought and how he saw the world, in particular how the concepts of the continuum was looked at. [4] "Basic to the progress of all science, he felt was an acceptable concept of continuity. It's nature and properties had always stimulated passionate controversy and great differences of opinion, though he was certain that the roots of were east to identify. Underlying the concept of the continuum, different features had always been stresses, but no exact or complete definition had ever been given. He assigned original blame to the Greeks, who had been the first to study the problem but in such ambiguous terms that myriad interpretations were left open to later doxographers and commentators. For example, Cantor believed that Aristotle and Epicurus represented polar opposites on the subject of the continuum and its consistency. On the one hand, Aristotle and his followers believed in a continuum composed of parts which were divisible without limit. Epicurus by contrast, developed the atomist position and regarded the continuum as somehow synthesized from atom which were imagined as finite entities." (Dauben)

Cantor did not agree with those who wanted a middle ground between the two, though at the end though Cantor would have been loath to agree, Cantor ultimately was closer to Aristotle than he was to Epicurus, though ultimately he agreed with neither. He for example did not 
agree with Aristotle's viewpoint that infinite numbers could "annihilate" finite numbers.

Cantor's proof is widely accepted, but there are always those dissenters though few in number who do not agree with Cantor's proof. [5] "Mathematicians feel that mathematical existence is established only when one of the objects whose existence is in question is actually constructed and exhibited. The above proof does not establish the existence of transcendental numbers by producing a specific example off such a number." (Eves). Transcendental numbers are non algebraic numbers. Though Cantor did not come up with such a number his theory was proved in a logical and systematic manner. To understand Cantor's logic it would be wise to read the book by the Kaplan couple entitled, "The art of the Infinite."

\section{Is Infinity Practical Outside Pure Mathematics}

Cantor might have came out with an acceptable proof of infinity, one should read Chapter nine of the book, "The Art of the Infinite". At the beginning of the Chapter the authors quote Galileo, [3] "In 1638 Galileo argued that "equal", "greater", and "less" can't apply to infinite quantities because a line segment contains an infinity of points, so a longer line segment would have to contain more than that infinity, which is impossible." Cantor would disprove this line of thinking by using set theory, sets of infinity within infinity, but infinity posses huge dilemma's outside pure mathematics that Galileo perhaps was possibly anticipating, maybe often, equal, greater and less cannot apply to 'infinite' quantities.

A twelve year old or even a six year old with reasonable mathematical abilities understands that numbers go on forever and any number followed by a decimal also goes on for ever, for example we can have

$0.134 . \cdots 9 \cdots \mathrm{n} \cdots$ just as numbers run from $1,2,3 \cdots \mathrm{n} \cdots$, $\mathrm{n}$ being a very large number. Technically speaking, a radius of a circle can for example be $1 \mathrm{~m}, 1.5 \mathrm{~m}, 1.5333$ $\mathrm{m}, 1.533336777 \cdots \mathrm{m}$ depending on the accuracy of our measuring instruments, what is important is that it is possible. Therefore $r$ follows the logic established by Cantor, $r$ can be divided into infinite parts after the decimal for example. The radius say it falls between 1 and $2 \mathrm{~m}$ or $\mathrm{cm}$, it can be for exams $1.5 \mathrm{~cm}$ or $1.59 \mathrm{~cm}$ or $1.599 \cdots \mathrm{cm}$, this cannot be debatable.

Throwing a stone in the air, it will return to the ground. [6] We can for example with proper instruments know how far the stone is from the ground as illustrated in Figure intro.

Does the concept of infinity hold in the example of the stone, say the stone is of the ground for $\mathrm{n}$ seconds. The stone after all cannot logically be of the ground for ever,

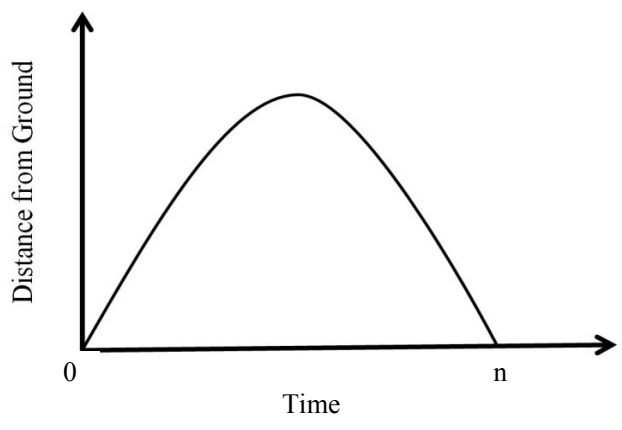

Figure intro: Throwing stone from ground.

there is the force of gravity therefore $n$, the number of seconds that the stone is off the ground cannot be forever whilst the radius of a circle can logically be infinitely large or at the least as big as the universe, a stone however will normally come down in a matter of seconds, therefore logically speaking $\mathrm{n}$ cannot be infinitely large. Let us say that $\mathrm{n}$ is 4 seconds, therefore at anytime between 0 and 4 seconds the stone is off the ground and in motion. It follows that at 3 seconds the stone is still in motion even at 3.9 seconds or $3.999,3.99999$, or $3.999 \cdots$ seconds the stone is still in motion. It follows that Cantor's logic holds with the stone been thrown in the air. The stone is in constant motion until it hits the ground after $\mathrm{n}$ seconds, however each second that it is in the air can be split into as many parts as instrumentation allows us to measure, at 2.5 seconds the stone is off the ground, at 2.51 seconds the stone is of the ground, at $2.511,2.5111,2.51111 \ldots$ seconds the stone is still of the ground and seconds logically can be split into infinite parts. Time is truly a continuous phenomenon and like distance can be broken into infinite parts. It is the quality of the continuous that allowed calculus to be discovered. Reading Dewdney's book “A Mathematical Mystery Tour", one will understand the logic of how Leibniz discovered calculus. Some claim Newton discovered calculus but hid it for twenty years, but that is for Newton's defenders, why hide your work and when somebody else publishes their work claim to already know that. When Leibniz discovered calculus he could do so because of the nature of the continuous.

By seeing how far the stone is off the ground, with calculus we can see the rate of change, that is to say the velocity of the stone in this case. It works perfectly because time follows Cantor's logic and can be divided into infinite parts. Therefore at 2.2 seconds we can know the rate of change of the stone, to be more specific the velocity as well as at 2.22 or 2.2267788 seconds we can know the rate of change of the stone because of the nature of time 2.2267788 seconds does exist logically and practically. In fact differential calculus is very important in today's world. [7] "The new calculus came to be applied to just about every conceivable type of motion or change 
in the physical world. Often mathematicians or physicists would begin with an equation that involved differentials, moving by integration to an actual formula of position, ... Such equations called "differential equations", have dominated physics ever since. They appear in Schrodinger's equation of the hydrogen atom and in Einstein's theory of general relativity." (Dewdney)

The "new calculus" came to be applied to just about every conceivable type of motion or change because the changes being measured were/are seemingly instantaneous, this seemingly instantaneous change is supported by and itself supports Cantor's assertions on the concepts of the mathematical infinity. A stone thrown in the air and in 4 seconds hits the ground again it will be in motion in 2 seconds, it will be in motion in and at all parts between 2 and 3 seconds, because seconds can be broken into infinite parts. This seemingly instantaneous change depends on a large part on the unit.

\section{The Unit}

Cantor's abstract conceptualization of the infinite seemingly defended the non abstract concept of a stone thrown into the air. Truly understanding the abstract concept laid down by Cantor is itself stretching the imagination, but often it is easily applied in the real world. That between 0 and 1 there are infinite numbers as well between 0 and 0.1. Between each real number there is a set of infinite sets, countless, but can this affirmation always be applied in the real world.

Take a piece of wood say $10 \mathrm{~cm}$ wide, $10 \mathrm{~cm}$ high, and 3 meters long. It is cut into two pieces, each piece $10 \mathrm{~cm}$ wide, $10 \mathrm{~cm}$ high but different lengths. One is 2 meters long the others is 1 meter long. Using Cantor's abstract conceptualization that say there are infinite parts between 0 and 1, 0 and 2, and, 0 and 3, infinity is everywhere in Cantor's abstract, seemingly true with the variables such as time and distance but can it be true when we cut the two wood blocks above that have same height, and width but different lengths. Wood is basically made up of molecules. It follows that the larger block of wood has more molecules than the smaller block of wood, though the molecules are similar since the two blocks where originally one block that was cut into two blocks of varying sizes. The larger block has to have more molecules and they have to be a finite number, therefore Cantor's abstract conceptualizations do not hold in this case. You can not break the molecules into infinite parts, once the molecules are broken it is no longer wood.

The illustration above regarding wood does not follow Cantor's mathematical abstraction of infinity in two very specific ways:

1) With the wood the larger block has more parts, (the basic part being a molecule), than the smaller block;
2) Both blocks being built by molecules have finite parts that they can be broken down to.

The logic that there are infinite parts breaks down in the reality where molecules of wood are concerned for example.

\section{Why Cantor's Mathematical Infinity Often Fails in the Material}

Why Cantor's abstraction often fails is because of the nature of the material, for this it will be easier understand if we go and understand an acceptable scientific theory concerning the beginnings of the universe, we must go to the "Big Bang" theory. "The Big Bang theory is an effort to explain what happened at the very beginning of our universe. Discoveries in astronomy and physics have shown beyond a reasonable doubt that our universe did in fact have a beginning. Prior to that moment there was nothing; during and after that moment there was something: our universe [8] ... According to the standard theory, our universe sprang into existence as 'singularity' around 13.7 billion years ago ... The pressure is thought to be so intense that finite matter is actually squished into infinite density." (All About Science). The usually cliché is that the universe started as a small dot, what is important is the matter was finite contained in that small dot. There is so much matter in the universe, the matter might be transformed but it remains finite. One of the first real lessons one learns in science is that energy cannot be destroyed only transformed, you cannot increase the amount of energy in the universe, because it is finite, all those many billions of years ago contained in a small "dot".

Assuming all the energy in the universe amounted to N, you therefore cannot have more than or less than $\mathrm{N}$ amounts of energy. However one can count to $\mathrm{N}+1$, or $\mathrm{N}+100$, but $\mathrm{N}+1$ and $\mathrm{N}+100$ are just abstractions, the universe cannot have that amount of energy, there is a limit, just as a piece of wood is limited into how much it can be broken down, one will end up smashing molecules and atoms and getting completely different materials.

The universe might be expanding but it has finite energy and therefore how much matter it can contain and matter cannot always be broken into infinite parts. Numbers were invented by human beings to aide counting and measuring, that we can find relationships in numbers aides in the measuring process. But because of nature itself we cannot apply the same logic to every variable in those relationships. Some variables will follow the logic of the infinite as expressed in mathematics and developed by Cantor, variables like time, some will not, variables like wood.

Independent variables that follow Cantor's obvious 
logic, that is to say can be broken into infinite parts such as time, distance, been able to be broken into infinite parts can have change that is seemingly instantaneous in say differential calculus. Change from 3 seconds to 4 seconds for example is meaningless, because there is something occurring at 3.5 seconds, 3.6 seconds, 3.9 seconds, $3.99 \ldots$ seconds, the rate of change because of the nature of an independent variable such as time can be measured at exactly or near exactly 4 seconds.

What however is the rate of change say not if the independent variable is not so divisible, that is to say cannot be broken into infinite parts like time, or distance, what if the independent variable is addition of atoms of a certain element adding them from 3 to 4 . We cannot have $3.99 \ldots$ atoms, or 3.5 atoms it is illogical, Cantor's logic cannot hold, the instantaneous change has a different meaning, in the case of the atom because it is an indivisible variable, the instantaneous change is the resultant change from 3 atoms to 4 atoms.

In economics a major variable is labor, add so much labor and output increases by so much for example. Labor is not like time or liters that can be broken into infinite parts, a human is just that a human, we cannot have half a laborer, a laborer cannot be broken into parts let alone infinite parts. How then can the formulae/differential calculus for rate of change for labor output where the variable labor cannot be broken into infinite parts, be the same as when time is the independent variable, whilst time can be broken into infinite parts, it should be obvious that the differential calculus needs to be modified. The calculus invented by Gottfried Wilhelm Leibniz needs to be modified to deal with variables that cannot be broken into infinite parts. If the variable can be broken into infinite parts like time, there is without doubt no need to modify Leibniz's work. It is mandatory to make these changes otherwise independent variables be they be able to be broken into infinite parts or not are being treated the same, that is misguided logic.

Before moving further let us go back to the indivisible atom and indivisible laborer. Now there are people who will talk of atoms been divided and broken up, the atom bomb and nuclear power stations being the great examples, however when atoms are broken up they become different elements, in theory if one for example had a way to add or subtract protons, neutrons and electrons they could literally create water from uranium, gold from lead, but adding or subtracting protons, neutrons, and electrons creates a different isotope or element.

Returning to the indivisible laborer and atom, the calculus discovered by Leibniz has shortcomings if such variables were independent variables, however that cannot be true if they are dependent variables. If time is an independent variable for example and labor a dependent variable, the calculus of Leibniz must hold, because it will show a change at that particular moment. It is not illogical for example to say labor growth is predicted to grow at 1.5 laborers per day. True it is impossible to have 0.5 of a laborer, but one can understand that every 2 days 3 laborers are hired as the economy grows. It is the nature of the independent variable that is crucial in our debate. It is because a growth of 1.5 laborers per day on average is understandable, but what 1.5 laborers can produce is utter nonsense in the real material sense, the world, universe we exist in.

Clearly there are different classes of the continuity, a mathematical expression or equation can represent two classes of the continuity depending on the quality of the independent variable. Take an equation like $\mathrm{Y}=\mathrm{X}^{2}$, it is obviously continuous, for any number $\mathrm{X}$ no matter how large there is a $\mathrm{Y}$ value. However, if $\mathrm{X}$ represents a variable that can be divided like time and distance, it behaves very different to say if $\mathrm{X}$ represents labor or atoms, we shall discuss this further towards the end of the paper.

\section{The Rate of Change with Indivisible Variables: The Khumalo Derivative}

The equation $\mathrm{Y}=\mathrm{X}^{\mathrm{n}}$ illustrates a number relation, for every $\mathrm{X}, \mathrm{Y}$ increases to the power of $\mathrm{n}$ of $\mathrm{X}$. The derivative of this equation, $\mathrm{Y}=\mathrm{nX}^{\mathrm{n}-1}$, is itself a number relation and no more. It is a number relation that tells us the rate of change for $\mathrm{Y}$ for every value of $\mathrm{X}$ of the original equation, $\mathrm{Y}=\mathrm{X}^{\mathrm{n}}$. But we know that there are different classes of the continuous, the instantaneous change has different qualities, whilst some instantaneous changes are there at the moment say at 4 because of the nature of the continuous, it is not true for all, because we cannot have for example half a human being representing labor.

The Khumalo derivative is the modification of the Leibniz derivative, at this stage, strictly concerning itself with polynomials. It is a modification concerning the derivative when the independent variable cannot follow Cantor's logic like labor, labor cannot be broken into infinite parts, it is strictly a whole unit. A variable like labor can only be counted as natural numbers, whilst a variable like time can be split into infinite parts, and with a variable like time that can be split I to infinite parts, the Leibniz derivative needs no modification.

Therefore in the original paper where the Khumalo derivative appeared entitled [6] "Revisiting the Rate of Change", the author, though the mathematics was correct, was wrong to say in his conclusion that, "it is more prudent to use $\mathrm{f}_{\mathrm{k}}^{\prime}(\mathrm{X})$, it is correct", where $\mathrm{f}_{\mathrm{k}}^{\prime}(\mathrm{X})$ is the Khumalo derivative. Evidently with greater insight, $\mathrm{f}_{\mathrm{k}}^{\prime}(\mathrm{X})$ can only be used when the independent variable is indivisible at it's basic unit like labor, an atom, molecules, and in many instances capital. What is half a machine, let us say somebody owned a laundry business, 
what is half of a washing machine, it is then illogical to use the traditional derivative as discovered by Leibniz, the traditional derivative in symbolic form $\mathrm{dy} / \mathrm{dx}$ or $\mathrm{f}^{\prime}(\mathrm{X})$. Notation after all is a way for all to understand, one fixated with correct notation form is not likely to be a true scientist, will not discover laws of the material, that is more important than being fixated on correct notation, comma's, and semi colons, it does not make sense.

This paper was not written in order to lay out the Khumalo derivative, that paper has already been written, this paper is written in order for us to appreciate the concept of infinity and its limitations in science, the material world outside abstractions, especially in this context in economics. The Khumalo derivative is merely a modification of the Leibniz derivative calculus, merely to correct for indivisible independent variables, units. The Khumalo derivative is left as a number series but need not be so, as one will understand on further reading.

For example, we know from the paper [9] "Revisiting the Rate of Change", that the Khumalo derivative $\mathrm{f}_{\mathrm{k}}^{\prime}(\mathrm{X})=\mathrm{f}^{\prime}(\mathrm{X})-\mathrm{f}_{\mathrm{k}}^{\prime}\left(\mathrm{X}^{\mathrm{n}-1}\right)-\mathrm{R}$ to compensate for an independent variable being indivisible. $\mathrm{f}^{\prime}(\mathrm{X})$ being the traditional derivative. One truly gets different results. We can pick up any book on calculus to illustrate the point.

Take the function $f(X)=X^{3}-4 X^{2}+3 X+7$. Let us say that $X$ represents time and $f(X)$ an increasing phenomenon over time. What is the rate of change say at 5. Time can be divisible into infinite parts, it follows and is backed by Cantor's view of the infinite, of the continuous. The rate of change at 5 can be viewed as happening at 5 , the traditional view as expressed by Leibniz, the only way to solve this is of course using the traditional derivative, $\mathrm{f}^{\prime}(\mathrm{X})$. It follows that the relationship of numbers defining the rate of change, that is to be more scientific like, the derivative is $f^{\prime}(X)=3 X^{2}-8 X+3$. What is the rate of change at 5 seconds, it is $f^{\prime}(5)=$ $3(5)^{2}-8(5)+3=38$.

On the other hand, what if the function $\mathrm{f}(\mathrm{X})=\mathrm{X}^{3}-$ $4 X^{2}+3 X+7$, was changed so the $X$ represented labor and $\mathrm{f}(\mathrm{X})$ output in some production. What is the change of output at 5. Labor cannot be broken into infinite parts, the change logically can only be from $4-5$. We have to use the Khumalo derivative. $\mathrm{f}^{\prime}(\mathrm{X})$. From reading "Revisiting the Rate of Change", we know that for the above function, $\mathrm{f}_{\mathrm{k}}^{\prime}(\mathrm{X})$ is:

$$
\begin{aligned}
f_{k}^{\prime}(X) & =\left[3 X^{2}-3 X+1\right]-[8 X-4]+3 \\
& =3 X^{2}-11 X+8=28 .
\end{aligned}
$$

This is because:

$\mathrm{f}_{\mathrm{k}}^{\prime}(\mathrm{X})$ of $\mathrm{X}^{3}=3 \mathrm{X}^{2}-3 \mathrm{X}+1$

$f_{k}^{\prime}(X)$ of $4 X^{2}=4[2 X-1]=8 X-4$

The properties of labor and time are different, the differentiation cannot be the same, hopefully one can appreciate these differences, the rest of the acceptance is not scientific but ideological and this paper is not dealing with that.

\section{Understanding the Continuous, Understanding Classes of the Continuous}

Looking carefully at the khumalo derivative, kd, we notice it is a complex way of showing a simple logic, it is a relationships of number, and should be left as it is [9]. However, we will now try to explain the continuous further so that we understand that what the expression for the rate of change for one continuous variable cannot be the same as another, we have clearly identified two types of independent variables, ones that can be broken into infinite parts and others that just cannot as it will be meaningless. Logic must still apply in our analysis of variables.

Take Figure 1, of $Y=X^{2}$. It is obvious that $Y=X^{2}$ is a continuous function, it goes on forever, when $X=\infty$, then $Y=\infty^{2}$, if $\infty^{2}$ can exist or mean anything. The idea is that we understand that $\mathrm{Y}=\mathrm{X}^{2}$ is a continuous function by any standard of the word continuous. Figure 1, just goes up to $X=3$ because that is all we need for the purposes of these demonstration. When $X=3$, obviously $\mathrm{Y}=9$. Figure 2 shows the same function, $\mathrm{Y}=\mathrm{X}^{2}$, but it allows us to see only what is happening between $X=2$, and 3. We have not yet defined $\mathrm{X}, \mathrm{X}$ and $\mathrm{Y}$ can be any variables, obviously $\mathrm{X}$ being the independent variable. What happens when we define $X$ and $Y$ ?

Let $\mathrm{X}$ represent time and $\mathrm{Y}$ change in some phenomenon due to increasing time. Let us say $\mathrm{X}$ represents time in seconds, it could be hours or days, the properties of time will not change. This is represented by Figure 3 . We can know what is happening at 2.5 seconds, s.s5 seconds or even at 2.9 seconds. We merely look at calculus and we know the derivative for $\mathrm{Y}=\mathrm{X}^{2}$ is $\mathrm{Y}=2 \mathrm{X}$. At 2.25 the rate of change of the phenomenon that changes over time is $2(2.25)=4.5$, and at 2.9 it is 2

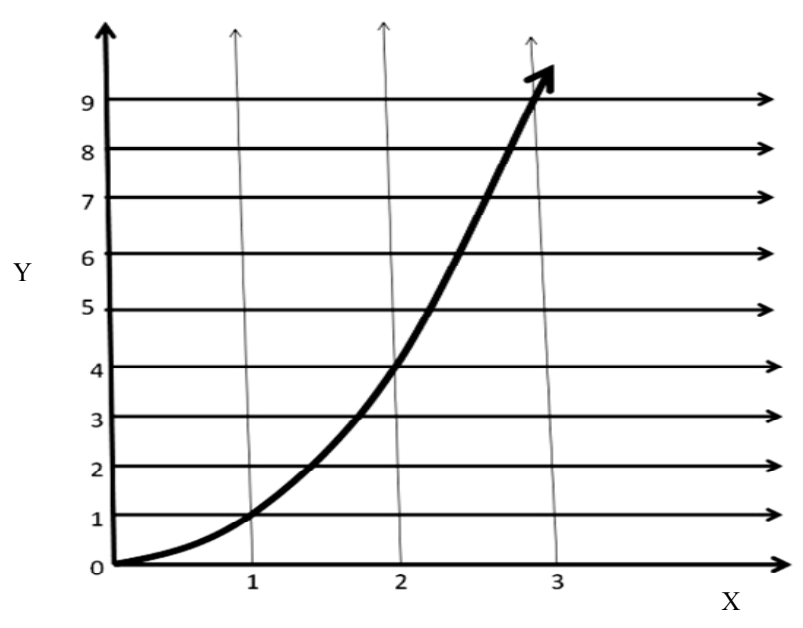

Figure 1. $Y=X^{2}$. 
$(2.9)=5.8$. That is the rate of change, the speed so to say. What if $X$ represented labor as in Figure 4?

We really do have a problem in logic, we cannot have 2.25 of a laborer, or 2.5 or 2.75 . We could just ignore this fact as most economists have done because of the battle is economics a science. To be a science one must use tools other scientists use even though the tools obviously sometimes do not make logical sense. This is the dilemma economists find themselves to be accepted, they must quote the "natural" sciences with no deviation lest a mathematician or physics says economics is not a science. But when economists look at a problem through scientific eyes, using tools of science, it is obvious that one cannot

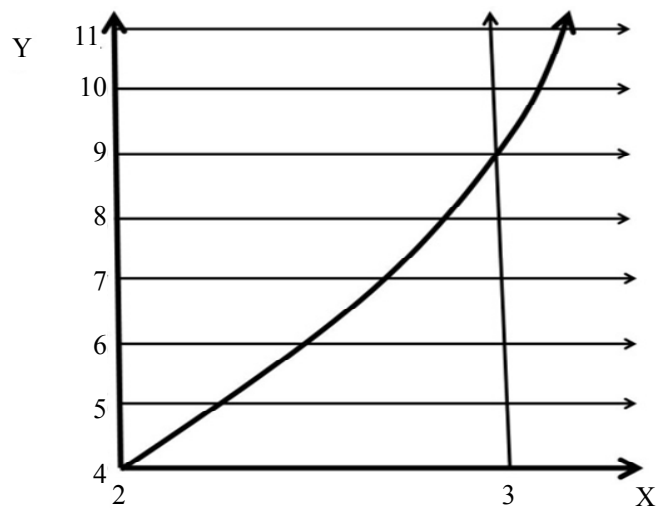

Figure 2. $Y=X^{2}$ : between 2 and 3 .

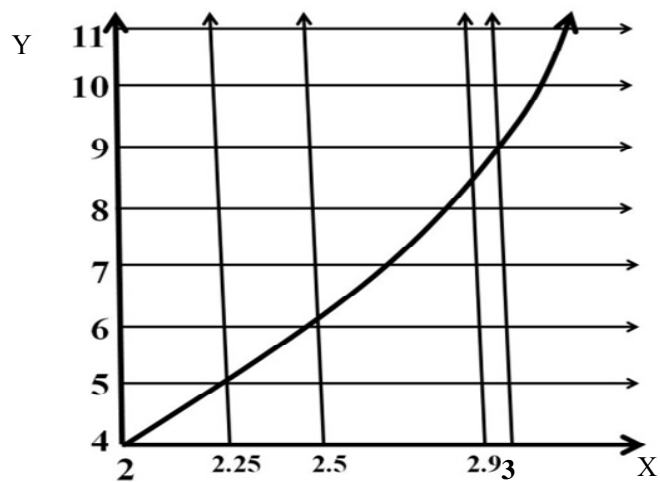

Figure 3. $Y=X^{2}$ : between 2 and 3 for $X=$ Time.

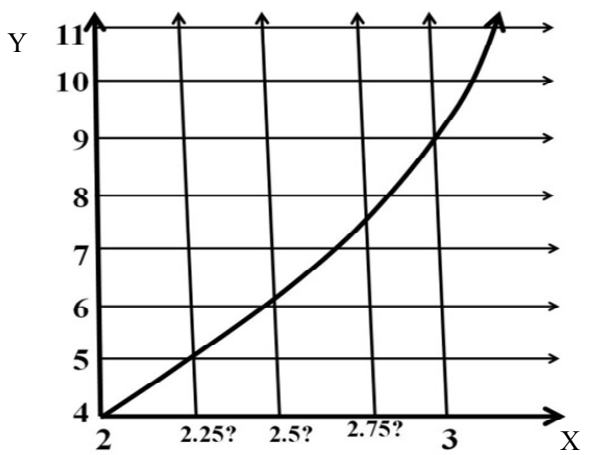

Figure 4. $Y=X^{2}$ : between 2 and 3 for $X=$ labour. treat time and labor the same for instance, though the relationship $\mathrm{Y}=\mathrm{X}^{2}$ remains continuous be $\mathrm{X}$ representing time or labor. Let us look at $\mathrm{Y}=\mathrm{X}^{2}$ again from a different perspective as illustrated in Figure 5.

Figure 5 is exactly the same $\mathrm{Y}=\mathrm{X}^{2}$ as illustrated in Figure 1 except that it has been graphed differently. Figure 6 is the imposition of Figure 1 on Figure 5, however it is Figure $\mathbf{5}$ that we are interested in. In Figure 5, it is understood from one's teen years that every point between say 1 and 2 represents 2 , any point greater than 1 but less than or equal to 2 , represents 2 . In the early years of one's mathematics we are taught there a different ways of showing the same information according to how one wants to present their illustration. One can go further and say there are different ways of showing

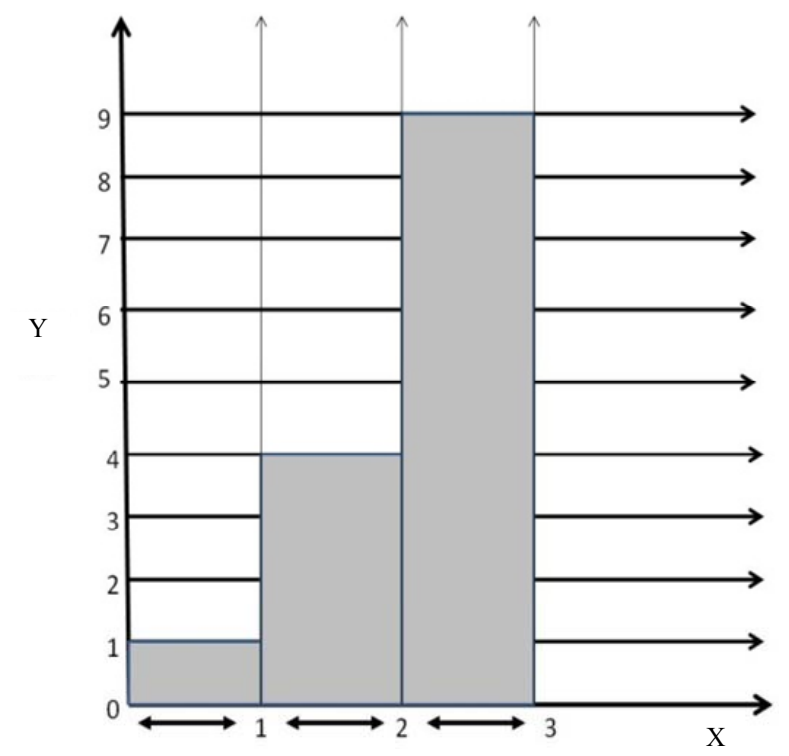

Figure 5. $Y=X^{2}$.

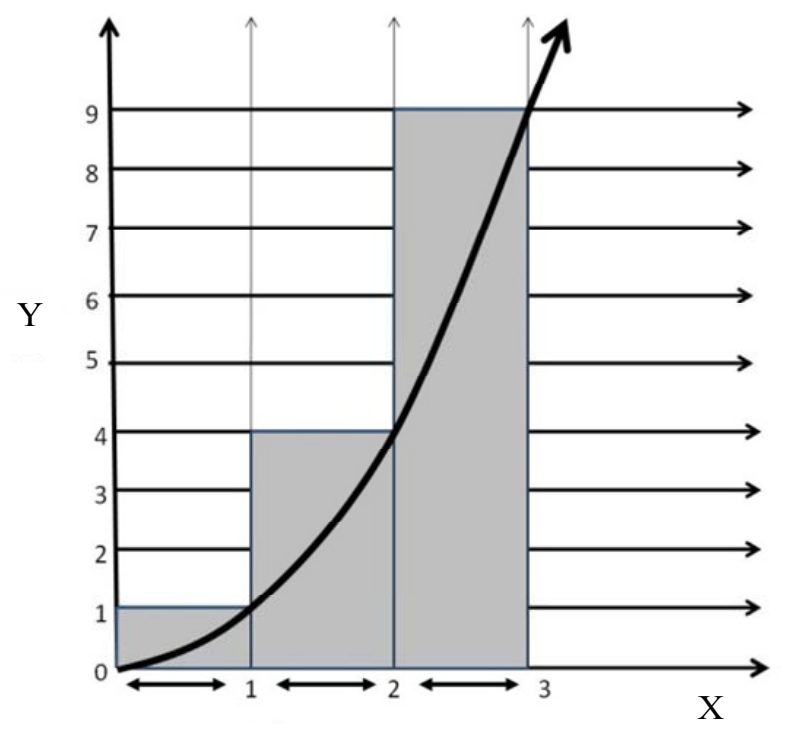

Figure 6. $Y=X^{2}$. 
information according to the characteristics of the independent variable. Figure 5 would suite representing labor as the independent variable because we cannot have say 1.5 laborers. The next number of laborers after 1 is 2 , and Figure 5 clearly shows that. It looks more scientific to use Figure 1, but it is scientifically wrong to use Figure 1 and then interpret it like time, using Figure 5 one cannot make that mistake, if however one understands the different classes of the infinite, even using Figure 1 they will not make that mistake. We can understand this phenomenon better by looking at Figure 7.

Figure 7 shows what is happening between 2 and 3 when $\mathrm{X}$ represents an independent variable like labor, or variables with the same kind of continuous, that is to say they cannot be divided into infinite parts. Between 2 and 3 there is nothing, because nothing can exist, we cannot have 2.4 laborers, it is a factual impossibility, that is why after 2, and less than or equal to 3 , all values are nine. Putting a line is merely connecting the points as illustrated in Figure 6, but, one must understand the nature of the variable. One can see the point been laid across even in Figure 7, after 2 all points are 9, but there is a line that can be placed uniting 2 and 3 , it is just a line depending on how one wants to present their information. The line is meaningless until it is equal to 9 or 4 , it is for illustrative purposes. The mistake made is when one believes there is something there and starts finding the rate of change for 2.6 laborers, most unscientific.

\section{Classes of the Continuous}

To understand what the khumalo derivative, $\mathrm{kd}$, really represents, and what is meant when one says it can only be used with a certain class of variables, with variables that cannot be divided into infinite parts but can be expressed in an infinite series like when we take $\mathrm{X}$ as labor in the equation $Y=X^{2}$. We need to modify Figure 7 to Figure 8. What is the rate of change from 2 to 3 in Figure 7 or Figure 5, obviously there is no tangent to guide us, and a tangent cannot be found, it is impossible.

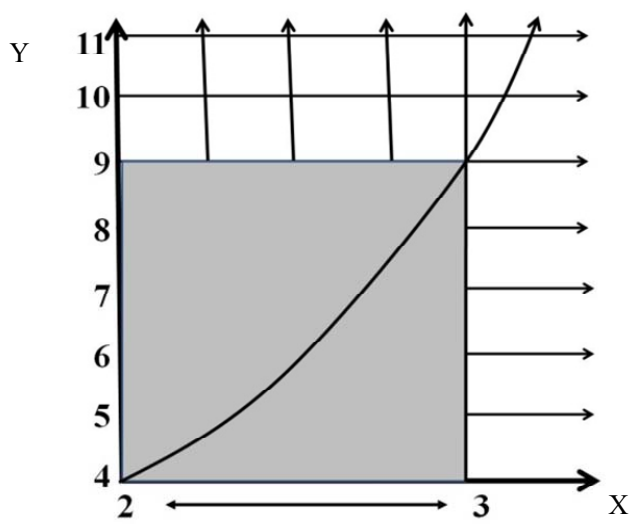

Figure 7. $Y=X^{2}$ : between 2 and 3 for $X=$ labour.
Looking at Figure 8 we see the rate of change, and it is instantaneous but, we can only understand this instantaneousness from the nature of the graph. Every value greater than 2 but less than or equal to 3 is 9 . That is to say $2<\mathrm{X} \leq 3=9$. This is because anything after 2 is 3 . But in reality there is nothing in-between 2 and 3 hence the notation $2<\mathrm{X} \leq 3$ is itself misleading. To understand these we will have to look at the set of numbers, basic number lines should help us understand. The mind is also aided by visualization, visualization can help simplify what otherwise would be complex explanations. It is how we present say, a number line that affects in some part our understanding of numbers and therefore of infinity. Take Figure 9 as an example.

Figure 9 shows a number line showing positive integers, 0 has been included. Figure 9 shows the number line up to 8 however we know it goes all the way into infinity. When we look at the number line we see there are spaces between every number, and we know that there are fractions and that the fractions fill up those spaces. In fact in those spaces there are infinite sets.

Figure 10 illustrates what is happening in between 2 and 3 on the number line.

As we can see from Figure 10, in between 2 and 3 there infinite sets, the number line can continuously be broken, this is infinity in mathematics as we generally understand it, it is this concept that backs up the work of mathematical models in most sciences. Hence we have a second for example that can be broken into infinite parts, we have a meter that also can be broken into infinite parts. What of those variables that can logically not agree

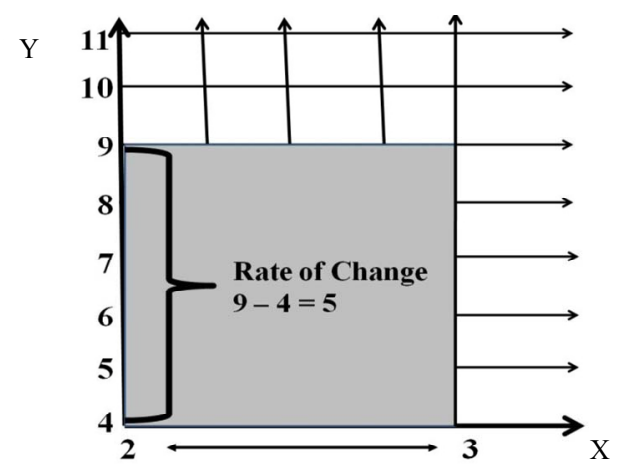

Figure 8. $Y=X^{2}$ : between 2 and 3 for $X=$ labour.

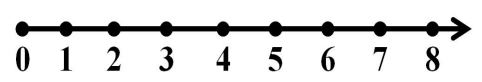

Figure 9. Set of positive integers.

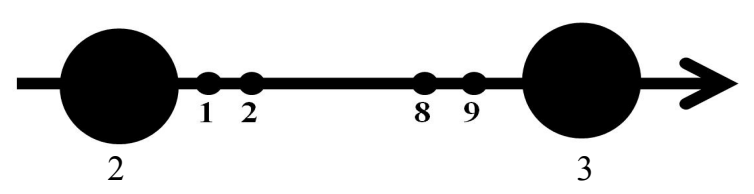

Figure 10. Set between 2 and 3. 
with the number line as represented in Figure 10, though Figure $\mathbf{1 0}$ is true. Our visualization of the number line does not allow us to understand that the number line above as represented in Figures $\mathbf{9}$ and $\mathbf{1 0}$ is only one class of the infinite, what if we changed Figures $\mathbf{1 0}$ and 11, hopefully now we can understand another class of infinity, mathematics must also follow reality, reality must define mathematics not the other way round.

Figure 11 shows part of a number line where there are no spaces between 2 and 3 , in this number line you cannot have 2.2 or 2.9, you have 2 and 3 . Figure 12 shows the number line for this class of numbers with this property. There are no spaces between the integers. Figures 11 and 12 visually illustrate what is happening in Figures 5 and 8 . Figures 11 and 12 illustrate the reality of certain variables like labor that cannot be divided into infinite parts. Because the number line in Figures 11 and 12 cannot be broken into infinite parts, the calculus developed by Leibniz cannot possibly work because there are no tangents that can exist as illustrated in Figures 58.

Therefore returning to Figure 8 above, the rate of change for adding one more laborer at 2 to get to three is 5 given the function $Y=X^{2}$. Surely no sensible answer exists except that, and the Leibniz derivative would give you 6 , that is clearly wrong from the clarification of the infinite given above. In the real world, there are at least two classes of the infinite as represented by Figure 9 and 12 , one can only hope that this paper has shed some light on this interesting subject matter. It does not matter what anybody says, or what ideology they are infested with, you cannot break labor into infinite parts though you can do it with time and distance.

Having established the fact that there are two classes of the infinite known to mankind we can name them. The first class as expressed by Cantor and Indian Mathematicians centuries before him including Bhaskara who predates Leibniz by 500 years. Bhaskara used a form of calculus long before Leibniz. The class of infinity as

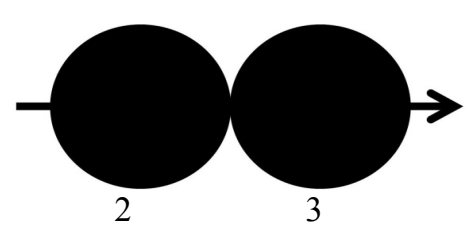

Figure 11. Set between 2 and 3.

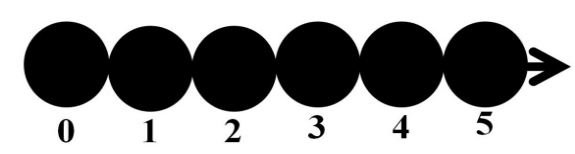

Figure 12. Another class of positive integers. known by Cantor, Leibniz and Bhaskara would be the infinite of the one dimensional, like time, and distance. One would use the differential as illustrated by Leibniz.

The second class of the infinite covers the three dimensional, these variables can not be broken into infinite parts. One would use a derivative as illustrated by Bhekuzulu Khumalo.

\section{Trans-Dimensional Mathematics}

Now we know the differential behaves differently according to the dimension of independent variable, a 3 dimensional independent variable has a different rate of change to a 1 dimensional independent variable. Incidentally, for those who have read knowledge economics and understood the properties of knowledge, they will understand that the rate of change of adding units if similar quality concerning knowledge there is no change at all. One must clearly understand that the rate of change is affected by the characteristics of the independent variable; this is the beginning of understanding of what is called trans-dimensional mathematics, a courtesy of studying knowledge economics in the proper context.

The above paper has outlined the beginnings of transdimensional mathematics. We must modify the differential for changes in dimensions of the independent variable.

- Leibniz differential for 1 dimensional independent variables.

- Khumalo differential for 3 dimensional independent variables.

- ? for 4 dimensional independent variables

- ? for $5-\infty$ dimensional variables.

Understanding the trans-dimensional properties and the differential for example, we understand that the higher the dimension of an independent variable the rate of change slows down, the rate of change is not as great as independent variables with lower dimensions. The derivative created by Leibniz and Bhaskara for example is not suitable for 3 dimensional independent variables, but then it follows the khumalo differentiation itself though suitable for 3 dimensional independent variables is not suitable for 4 dimensional independent variables. Therefore if one was to study a 4 dimensional phenomenon, they would need to modify the Leibniz differentiation for 4 dimensional phenomenon. Therefore to study existence as a whole, the existence we know, the 4 dimensional universe of matter and time, one will never get the correct answer and proper analysis unless they modify derivative mathematics to suite 4 dimensional phenomenon, and they will find the changes are not as rapid as they believe the changes are. But one can use the derivative for lesser dimensions to study phenomenon within the 4 dimensions we exist in. 
The wonder of knowledge is that it exhibits properties of 1 dimension, 3 dimensions and infinite dimensions. But mostly it exhibits properties of 3 dimensions and infinite dimensions. It exhibits properties of infinite dimensions in that we can add the same knowledge and the result will be the same knowledge. I am assuming that is infinite dimensions because there is no change therefore $\mathrm{dx} / \mathrm{dy}=0$ no matter how much similar knowledge you add from different people. It is the study of knowledge economics that has led us to understanding the need for different derivatives depending on the nature of the independent variable, and we have called these qualities trans-dimensional mathematics.

In mathematical notation, for those who prefer mathematics, we take a polynomial function $\mathrm{f}(\mathrm{x})=\mathrm{X}^{\mathrm{n}}$, then derivative is:

$$
\mathrm{f}^{\prime}(\mathrm{X})=\mathrm{nX}^{\mathrm{n}-1}-\mathrm{R}_{\mathrm{i}}
$$

where $\mathrm{R}$ is the residue $\mathrm{i}$ the number of dimensions a variable has $\mathrm{nX}^{\mathrm{n}-1}$ the core derivative.

- For 1 dimensional independent variables $\mathrm{R}_{\mathrm{i}}=\mathrm{R}_{1}=0$ therefore the derivative is:

$\mathrm{f}^{\prime}(\mathrm{X})=\mathrm{nX}^{\mathrm{n}-1}$ and this is equal to derivative of Bhaskara and Leibniz.

- For 3 dimensional independent variables $\mathrm{R}_{\mathrm{i}}=\mathrm{R}_{3}=$ $\mathrm{f}_{\mathrm{k}}^{\prime}\left(\mathrm{X}^{\mathrm{n}-1}\right)-\mathrm{R}$, therefore the derivative is:

$$
\mathrm{f}_{3}^{\prime}=\mathrm{f}_{\mathrm{k}}^{\prime}=\mathrm{nX}^{\mathrm{n}-1}-\mathrm{f}_{\mathrm{k}}^{\prime}\left(\mathrm{X}^{\mathrm{n}-1}\right)-\mathrm{R}
$$

where $\mathrm{f}_{3}^{\prime}=$ derivative of third dimensional independent variable or $f_{k}^{\prime}$ the Khumalo derivative.

- For an independent variable with infinite dimensions $\mathrm{R}_{\infty}=\mathrm{f}^{\prime}(\mathrm{X})$ therefore the derivative is: $\mathrm{f}_{*}^{\prime}=\mathrm{nX}^{\mathrm{n}-1}-\mathrm{nX}^{\mathrm{n}-1}=0$, there is no change.

- For a 4 dimensional independent variable the derivative would be expressed as $\mathrm{f}_{4}^{\prime}$ and this derivative has not been discovered. The residual would be described as $\mathrm{R}_{4}$.

It should be clear that the statement $f_{i}^{\prime}$ and $R_{i}$, the $i$ in this case stands for the dimension of the independent variable. The independent variable for example is $f_{5}^{\prime}$ it follows the residual will be symbolized as $R_{5}$ and the independent variable is of 5 dimensions, and so on.

One can be sure that the same rules would apply to integration, but that has not been analyzed further.

\section{What Is Trans-Dimensional Mathematics}

Laid out above is the basic premise of transdimensional maths, transdimensional mathematics can be very simply defined as study of mathematics in different dimensions and comparing the results. It is clear from above that with increase of dimensions, the rate of change actually slows down, it has been proved. But there is a simple tool to prove it, take knowledge, if people know the same thing, there is no increase in knowledge when adding up knowledge, however, if they know different things, when you add up knowledge there is an increase in knowledge, because in one instance knowledge is behaving as a variable with infinite dimensions, therefore $1+1=1$, meaning no rate of change, and in the other instance knowledge is behaving as a three dimensional variable, less than infinite dimensions, and $1+1+2$ meaning there is a rate of change.

Obviously above it is only the foundation there is still much work, we still need to calculate three dimensional variable derivative using logarithmic functions, exponential functions, as well as all the other functions, as well as working on the theoretical base of the integral function as dimensions get higher.

Hopefully it is understood, the higher the dimension of an independent variable, the smaller the rate of change, the smaller the derivative, even if the equation is exactly the same.

When considering transdimensional mathematics it will be easier, (talking about future as the discipline is in its very infancy), it will be easier to consider the $Đ$ factor. The $\mathrm{Ð}$ factor can be considered as the quotient that determines the rate of change as we move to higher dimensions. Once the $Ð$ factor can be found we can find the rate of change at any dimension. In mathematical notation the $Ð$ factor can be expressed as:

$$
\mathrm{f}_{\mathrm{n}}^{\prime}(\mathrm{X})=\mathrm{Df}_{\mathrm{n}-1}^{\prime}(\mathrm{X})
$$

where

$$
0<\mathrm{Ð}<1
$$

There are two scenario's of Đ's possible behaviour, $Đ$ factor simply being the dimension factor. The first possibility is that $Đ$ is a constant, a constant like say $\pi$.

Secondly $Ð$ could be decreasing at a predictable rate, this is because there must be a time when $Ð$ is zero, because at infinite dimensions the rate of change is zero. In this scenario $Đ$ can be notational represented as:

$$
0 \leq \mathrm{\oplus}<1
$$

$Ð$ factor however will be explored in future mathematical papers, this is an economics paper.

\section{Mathematics and Economics}

This problem came about because of analyzing knowledge as a commodity, a field I call knowledge economics. Having given knowledge a unit, the knowl, in order to study it in an appropriate scientific manner, one finds knowledge behaving as a 3 dimensional variable and a variable with infinite dimensions, this brought about the concerns about infinity. Economics is a science and should be treated as such. This problem would never have been found without treating economics as a science.

As economics becomes and aspires to be more scientific, mathematics will be used more and more as a sup- 
porter of arguments. There is the premise by some that if you can not measure it, it is not science. But as economists we need to be careful that mathematics does not obscure economic theory and more importantly that mathematics is not used wrongly just for the sake of making economics look scientific, it already is scientific enough. Economists like Hayek rightly did not trust the overuse of mathematics in economics.

Mathematics does not make anything more scientific or less scientific. Science is merely the demonstration of the laws of existence, science as defined by dictionary.com, "knowledge, as of facts or principles; knowledge gained by systematic study". Therefore it is a misguided concept that mathematics makes something more scientific, especially when it is used wrongly. But this does not mean there is no room for mathematics, usually to solve a problem and to have a definite prove, mathematics will be always crucial. Misuse of mathematics include saying from the blue that stocks behave like gases and using those equations for gases to predict the stock market, what is the assumption behind saying stocks behave like gases. Would it not be better to find out how stocks behave in general and then find an equation that defines that, that stocks are a result of human behavior, it means their behavior is natural and have their own laws that determine them rather than laws specifically for gases, we are just been lazy.

Economics is essentially about demand and supply, mathematics will always be needed to give us a view of complex demand and supply conditions, and to allow us a guide of when supply will stop flowing and when demand will slow down given certain prices in the market.

Dealing with the concepts laid out in this paper, the derivative is concerned with marginal concepts in economics, and these can be considered the basis of modern scientific economic theory by many. This paper should show that depending on the dimension of the independent variable, the derivative has to be altered, therefore the marginal value will be affected. If labor for example is the independent variable, the marginal increase given the same function will not be the same as if time was the independent variable. Therefore the mathematics presented in this paper should prove crucial to economics in the long run.

However, it must always be remembered that mathematics in economics can give greater accuracy, but can never be dead accurate, it gives us a trend, and trends are more important to economics as they give the general direction of the economy. Trying to be super accurate might end up in a model being too complex as there would be too many variables to include. What we need as economists is an accurate trend rather than a dead on figure, and using mathematics that suites the occasion will lead to more accurate trends.

\section{Economics Is a Science}

When treated properly I hope I have proved to many naysayers that economics is indeed a science. This problem of the dimension of a variable was discovered whilst studying and researching knowledge economics. Though discovered whilst researching knowledge economics, this mathematics is applicable to all sciences and fields that deal with mathematics. Economics has given back to science as a whole.

\section{Thanks}

Thanks to Guido Travaglini whose encouragement led me to show the whole series of what I called the Khumalo derivative, he immediately understood what the theory being postulated was all about.

\section{Books of Influence in Paper}

There are of course other writings that have directly helped in this paper though not quoted directly including [10] Benacerraf's Philosophy of Mathematics, [11] Faticoni's mathematics of infinity, [12] Dantzig's Number: Language of Science, [13] Zaslavsky's Africa counts, and, [14] Benson's moment of proof.

\section{REFERENCES}

[1] J. Mazur, "Euclid in the Rainforest: Discovering Universal Truth in Logic and Math," Penguin Books, New York, 2005.

[2] D. J. Struik, "A Concise History of Mathematics," Dover Publications, New York, 1967.

[3] R. Kaplan and E. Kaplan, "The Art of the Infinite: The Pleasures of Mathematics," Oxford University Press, New York, 2003.

[4] J. W. Dauben, "Georg Cantor: His Mathematics and Philosophy of the Infinite," Princeton University Press, New Jersey, 1979.

[5] H. Eves, "Foundations and Fundamental Concepts of Mathematics," PWS-Kent Publishing Company, Boston, 1990.

[6] B. Khumalo, "Revisiting the Derivative: Implications on the Rate of Change Analysis," 2009. http://www.repec.org./

[7] A. K. Dewdneyy, "Discovering the Truth and Beauty of the Cosmos: A Mathematical Mystery Tour," John Wiley \& Sons, New York, 1999.

[8] All About Science, "Big Bang Theory," 2009. http://www.allaboutscience.com/

[9] B. Khumalo, "The Concept of the Mathematical Infinity and Economics (Research Note)," International Advances in Research Economics, Vol. 17, No. 4, 2011, pp. 484 485

[10] P. Benacerraf and H. Putnam, "Philosophy of Mathematics: Selected Readings," Cambridge University Press, 
New York, 1983.

[11] T. G. Faticoni, "The Mathematics of Infinity: A Guide to Great Ideas,” John Wiley \& Sons, New Jersey, 2006.

[12] T. Dantzig, "Number: The Language of Science Pearson Education," New York, 2005.
[13] C. Zaslavsky, "Africa Counts: Number and Pattern in African Culture," Lawrence Hill Books, Chicago, 1973.

[14] D. C. Benson, "The Moment of Proof: Mathematical Epiphanies," Oxford University Press, New York, 1999. 